\title{
SERVICIOS PÚBLICOS DOMICILIARIOS: ¿DERECHOS HUMANOS O PRODUCTOS PARA LA VENTA? CASO MEDELLÍN.
}

HOME UTILITIES: ARE THEY HUMAN RIGHTS OR PRODUCTS FOR SALE? THE MEDELLIN CASE.

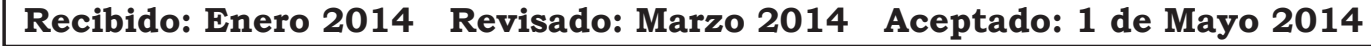

Por: Fabián Alirio Mazo Elorza ${ }^{1}$.

\section{RESUMEN.}

El presente artículo presenta los resultado de investigación encaminada a demostrar y visibilizar, en la ciudad de Medellín, las múltiples afectaciones a personas por la ausencia de servicios públicos domiciliarios en sus viviendas, aún y la capacidad técnica, operativa y financiera de la empresa prestadora de servicios en la ciudad: Empresa Públicas de Medellín (Epm E.S.P), lo que supone una inadecuada lectura de la realidad de la ciudad, principalmente en las zonas periféricas y zonas de alto riesgo. Esta situación lleva a que los ciudadanos recurran a las vías judiciales o a las vías de hecho para proveerse del servicio y en cualquiera de las dos vías se termina por generarle una serie de dificultades de orden jurídico, social y económico al Municipio que es, según la Ley 142 de 1994, el responsable de garantizar el derecho a los servicios públicos domiciliarios; situaciones que pudiesen contar con una solución más adecuada en tanto que ajustada a las realidad de la ciudad.

\section{PALABRAS CLAVE.}

Servicios públicos domiciliarios, Empresas Públicas de Medellín, Zonas de alto riesgo, Plan de Ordenamiento Territorial POT, Mínimo Vital.

\section{ABSTRACT.}

This current paper introduces the research results aiming at demonstrating and identifying, in the city of Medellin, the multiple effects to people by the lack of home utilities, still and the technical, operational, and financial capacities of the company providing services in the city: Public Companies of Medellin (EPM -ESP), which supposes an inadequate reading of the reality of the city, mainly on the outskirts and high-risk areas. This situation makes the citizens resort to the judicial routes or assault and battery in order for them to provide with the service, and in either of the two routes, a series of legal, social, and economic difficulties are derived, which becomes a problem to the Municipality, which according to the Law 142 as of 1994, is responsible for ensuring the people the right to home utilities, situations which could have a more adequate solution to the extent that it can be adjusted to the reality of the city.

\section{KEY WORDS.}

Home Utilities; Public Companies of Medellin -EPM-; High Risk Zones; Territorial Ordering Plan -TOP-, and Minimum Living Standard.

\footnotetext{
${ }^{1}$ Sociólogo, especialista en Gestión Urbana, Integrante del equipo de trabajo en Servicios públicos domiciliarios, minimos vitales de la Alcaldía de Medellín. Investigador académico. Medellín - Colombia. fabianmazo@hotmail. com,fabian.mazo@alcaldiademedellin.gov.co .
} 


\section{Introducción.}

En la última década se ha presentado a Medellín como líder en materia de Prestación de Servicios, en particular a EPM como la "mejor" empresa de servicios públicos del país y una de las mejores en América Latina, su presencia en muchas ciudades de Colombia (Bogotá, Bucaramanga, Pereira, Armenia, Manizales y todo el departamento de Antioquia) y en varios países de la región (Panamá, Puerto Rico, Guatemala, Salvador, México, Venezuela y Chile) consolidan esta imagen de la empresa lo que permite suponer que, siendo el municipio de Medellín su único propietario, este tema no genere mayor conflicto en la ciudad.

Por ello, la prestación de los Servicios Públicos Domiciliarios en Medellín se trata hoy, como un tema resuelto, debido a la fuerza y capacidad administrativa, técnica y financiera de la mayor empresa prestadora en la ciudad: Empresas Públicas de Medellin E.S.P. (Epm, 2014).

Ahora bien, el actual modelo de desarrollo económico, fundamentado a partir de la Constitución de 1991 y la legislación sobreviniente, se ha dirigido a favorecer los procesos de privatización de los servicios públicos, la creación de las empresas privadas y la posible entrada de multinacionales especializadas en este tipo de negocios, creando todo el andamiaje jurídico necesario para garantizarles la mayor sostenibilidad financiera posible al sistema empresarial de servicios públicos creado a partir de este modelo, ya sea de propiedad pública o privada.

Sin embargo, al momento de plantear el dilema de la necesidad de los Servicios Públicos Domiciliarios como elemento indispensable para el desarrollo humano, la obligación de prestar el servicio en las zonas de estratos más bajos y a las personas de más bajos recursos, frente a ese modelo de crecimiento económico y fortalecimiento empresarial de las empresas prestadoras de servicios públicos se recurre a crear un sistema de subsidios cruzados que permite el acceso de esta población a los Servicios Públicos Domiciliarios; esquema de subsidios que busca, fundamentalmente, otorgar un subsidio en las tarifas de los estratos 1, 2 y 3 que se compensan con contribuciones que hacen los estratos 5 y 6 y los sectores industriales y comerciales, esto como estrategia de asegurar que la población más vulnerable pueda tener acceso al servicio. Adicional a ello se mantiene en cabeza del Estado la responsabilidad de garantizar la prestación de los Servicios Públicos Domiciliarios a toda la población y además consagra en la Ley 142 de 1994, Ley de Servicios Públicos, que en aquellos lugares donde no haya un prestador que lleve los servicios es responsabilidad del Municipio llevarlos a toda la población.

Por otra parte, la Ley 388 (Ley 388, 1997), de ordenamiento territorial define que el perímetro sanitario no podrá ser inferior al perímetro urbano, lo que obliga a los municipios a garantizar la prestación del servicio en toda el área urbana de la localidad (Ley 388, 1997); a pesar de la norma dice que el perímetro sanitario no podrá ser inferior en la mayoría de las ciudades colombianas los Planes de Ordenamiento Territorial se han limitado a decir que son iguales, prohibiendo a las empresas prestadoras llevar el servicio por encima de este límite; además, también se fijan las zonas de alto riesgo geológico, retiros de quebradas y de vías principales y nacionales, retiros obligados a vías ferroviarias o del metro; en estos lugares tampoco se puede llevar, según las normas establecidas en el POT de la ciudad, (Acuerdo 046, 2006) la prestación de los Servicios Públicos Domiciliarios. 
Según cifras oficiales del Departamento Administrativo de Planeación en Medellín hay más de 180 mil hogares asentados en zonas de alto riesgo no recuperable, tal como se ve en el documento de diagnóstico para la revisión del POT; adicional a esto se tienen cifras muy diferentes por parte de EPM, (Epm, 2013) quien reconoce que se tienen en zonas de riesgo no recuperable 14.369 viviendas sin acueducto y 16.316 viviendas sin alcantarillado en estas mismas zonas; esto en el área urbana, situación que se agudiza si se suman las viviendas sin cobertura de estos servicios en el área rural.

Sin embargo, en Medellín la evidencia demuestra que aun en esos lugares con restricciones EPM ha prestado el servicio, pero hay algunas viviendas que están en esas mismas condiciones y la empresa les ha negado el servicio argumentando las restricciones de Ley; por otra parte, la población ante la necesidad del servicio, ya sea de agua y/o de energía han recurrido para obtener la prestación a dos mecanismos principales: por un lado a la conexión ilegal y fraudulenta, con los riesgos colaterales que esto trae de mayor inestabilidad del terreno por fugas de agua, incendios por malas conexiones de energía (Topalov, 2006), y por el otro, a la Acción de Tutela como mecanismo legal de obligar a EPM a instalar los servicios y vincular en la responsabilidad al municipio.

Los jueces de Tutela en repetidas sentencias han obligado a las empresas prestadoras a reconectar los servicios en los casos en que éstas habían suspendido o cortado el servicio por falta de pago, llegando casi a crear una jurisprudencia en esta materia, y han obligado a los municipios a llevar los servicios públicos aun a las zonas donde las normas imponen dichas restricciones, o brindan la alternativa de reubicar la población afectada toda vez que la Constitución Política fija los servicios públicos como uno de los fines esenciales del Estado.

Es ahí donde se genera una contradicción entre las mismas normas, más allá de una ambigüedad normativa: una Ley prohíbe la prestación de los servicios en zonas de alto riesgo (Ley 388 de 1997 y Ley 1450 de 2011, Acuerdo 46 de 2006) y otra obliga a llevarlos (Ley 142 de 1994 y Ley 143 de 1994). El verdadero dilema lo siente siempre la población que se asienta en esas zonas donde la norma prohíbe la prestación de los Servicios Públicos Domiciliarios, pero su condición de ser un bien esencial, absolutamente necesario para el desarrollo humano y para la vida misma, no les da más opción que buscar las formas posibles de acceder a ellos, ya sea por vias de hecho mediante las conexiones ilegales o mediante vias de derecho como la Acción de Tutela o las Acciones Populares.

Con todo lo anterior cabe preguntarse entonces: ¿Cómo puede el Estado garantizar la prestación de los servicios públicos, aun con las contradicciones normativas que se evidencian en Colombia? máxime cuando su modelo económico lo lleva no sólo a la privatización del servicio sino, además, a generar un modelo de ocupación del territorio que desconoce y excluye a sectores de la población.

Las normas urbanísticas, los instrumentos de planificación del territorio y las leyes que sobre servicios públicos existen o se expidan deben estar en armonía y coherencia las unas con las otras, de tal manera que se haga viable tanto la prestación del servicio en términos empresariales, que es como hasta ahora se ha pensado, como la garantía de los derechos fundamentales de la población. 
Abordar este tema obliga tener presente algunos elementos esenciales:

- La responsabilidad del estado en la prestación de los Servicios Públicos Domiciliarios.

- El criterio de sostenibilidad económica y financiera de las empresas prestadoras de Servicios Públicos Domiciliarios

- El proceso de poblamiento de las ciudades colombianas y particularmente en Medellin (Zambrano \& Bernardo, 1993)

Pues son estos criterios los que generan la contradicción normativa que ya se ha expuesto, y la forma de poblar la ciudad, sobre esta contradicción es que se pretende trabajar en el presente artículo con el siguiente objetivo general:

"Evidenciar las contradicciones normativas que hacen inviable la garantía de los Servicios Públicos Domiciliarios en una perspectiva de derechos humanos"

También se pretende, como objetivos específicos, los siguientes:

- Analizar la prestación de los Servicios Públicos Domiciliarios desde la perspectiva de los derechos humanos fundamentales

- Explorar las posibilidades de construir alguna alternativa de prestación de los Servicios Públicos Domiciliarios en las zonas de alto riesgo.

Para lograr estos objetivos se hizo un rastreo del estado del arte y principalmente de las sentencias que sobre el tema en particular ha emitido la Corte Constitucional.

Los servicios públicos domiciliarios no se han visto, en Medellín, como un problema de estudio, y sólo se tiene la referencia del drama de los desconectados que ponen sobre la agenda local algunas organizaciones sociales, pero hace falta que se empiece a dimensionar, más allá del asunto puntual de la desconexión, una perspectiva amplia de los derechos humanos que pasa necesariamente por armonizar la normatividad existente.

Alternativas de prestación de los servicios públicos desde una perspectiva de los derechos humanos que permita a todas las personas tener acceso a ellos.

\section{Metodologia.}

Los servicios públicos domiciliarios han sido vistos en direcciones opuestas; desde las empresas se ven como negocio, para el Estado se ven como un problema en algunos sectores puntuales, para los políticos como una oportunidad de hacer campaña y conseguir votos, y para la gente del común se ven como un elemento esencial del que se toma conciencia en el momento que se corta el suministro, mientras se goza y se disfruta pasan casi inadvertidos hasta el dia que llega la factura. Mientras esas visiones permanezcan y desde el Estado no se logra armonizar y crear una única visión de los derechos humanos que constituyen en sí mismos no será posible que se creen políticas públicas que garanticen a toda la población la prestación de los servicios públicos tal como hoy lo consagra la Ley 142 de 1994 en condiciones de cobertura, calidad y continuidad.

Por eso, este artículo, que busca evidenciar esas contradicciones normativas con el ánimo de que al hacer esto visible se pueda empezar a construir propuestas, 
desarrollará este artículo derivado de investigación, para su mayor comprensión, en cuatro partes así:

- Rastreo bibliográfico y normativo: En primer lugar algunos elementos generales sobre la concepción y la normatividad de los Servicios Públicos Domiciliarios, donde se empiezan a evidenciar algunas contradicciones en normas nacionales y locales y entre la normatividad de cada uno de los servicios. (Atehortua Rios, 2003)

- Análisis comparativo de las cifras y estadísticas oficiales: La segunda parte se centra en la problemática de la prestación de los servicios públicos domiciliarios en la ciudad de Medellin, las condiciones y características que tiene desde la prestación de acueductos comunitarios o veredales y desde el mayor prestador del servicio que es EPM.

- Análisis juridico y jurisprudencial, con enfoque de derechos: La tercera parte nos remite a la protección del derecho a los servicios públicos mediante la vía judicial y se hace una breve reseña de 4 sentencias y consideraciones de los magistrados para brindar esta protección mediante el mecanismo de Tutela. (Atehortua Rios, 2003)

- Conclusiones y Propuestas realizadas por los entes y propuestas emergentes: En la cuarta parte se enuncian las que han sido, hasta ahora, algunas alternativas a la mercantilización de los servicios públicos y se propone una visión desde la concepción de los derechos humanos. (McDonald \& Ruiters, 2012)

\section{Resultados y discusiones.}

$\underline{\text { Servicios Públicos Domiciliarios. }}$

La Ley 142 de 1994 o Ley de Servicios Públicos es la rectora de la prestación de los Servicios Públicos Domiciliarios en el país. Hasta antes de su expedición y antes de la Constitución de 1991 estos servicios eran prestados en su inmensa mayoría por empresas públicas del orden municipal, casi sin regulación o control por parte de la nación o del Estado más allá la vigilancia que hacían los Ministerios de Salud al tema de la calidad del agua y el Ministerio de Energía en cuanto a la generación y distribución de ésta. Regulación que se enfocó durante muchos años más a prevenir que el país se volviera ver sometido a un racionamiento como el que se vivió en 1992, que a garantizar la prestación del servicio a los sectores residenciales de cualquier estrato y ciudad

La Ley 142 abre la posibilidad de que ingresen empresas privadas al sector y que aquellas públicas se transformen en empresas Industriales y Comerciales del Estado, en empresas de carácter mixto o se privaticen; pero además se crea la Superintendencia de Servicios Públicos Domiciliarios, encargada del control y la vigilancia de las empresas prestadoras de servicios, y se crean además las comisiones de regulación, encargadas del diseño y regulación de la forma de prestación y el esquema tarifario en cada uno de los Servicios Públicos Domiciliarios así:

CRA: Comisión de Regulación de Agua Potable, Aseo y Saneamiento Básico

CREG: Comisión de Regulación de Energía y Gas.

CRC: Comisión de Regulación de Comunicaciones 
En el Numeral 5.1 del artículo 5 de la Ley 142 de 1994 dice textualmente:

Asegurar que se presten a sus habitantes, de manera eficiente, los servicios domiciliarios de acueducto, alcantarillado aseo, energía eléctrica, y telefonía pública básica conmutada, por empresas de servicios de carácter oficial, privado o mixto, o directamente por la administración central del respectivo municipio en los casos previstos en el articulo siguiente. (Ley 142, 1994)

El artículo 6 define que los municipios podrán prestar directamente los servicios de su competencia en los casos en que las características técnicas y "económicas", lo permitan y aconsejen.

En el artículo 14 define como Servicios Públicos Domiciliarios acueducto, alcantarillado, aseo, telefonía pública básica conmutada, energía eléctrica y gas combustible, tanto el servicios de gas domiciliario por redes como la distribución de gas licuado de petróleo (GLP). (Ley 142, 1994)

Así definido por las normas nacionales, los municipios están obligados a garantizar la prestación de los servicios públicos, principalmente aquellos definidos en el artículo 5 de la Ley 142; amparados en esta obligación legal se ha tutelado el derecho a los servicios públicos en las diferentes sentencias en las que la Corte Constitucional ha reconocido este derecho a la población.

Por otra parte, la configuración física del territorio, especialmente en Medellín, por ser un valle estrecho rodeado de una serie de montañas, que los geólogos han dado en denominar como dunitas, hacen que sus laderas sean empinadas e inestables, lo que genera que existan una gran cantidad de áreas que se consideran de alto riesgo geológico, es decir, zonas en las cuales las normas prohíben la construcción y ubicación de población en forma permanente, son zonas prohibidas para los procesos de urbanización.

Este fenómeno se presenta en varias regiones del país, el riesgo de deslizamientos, avenidas torrenciales, desbordamientos de rios y quebradas se vuelven una característica nacional, por lo que desde 1997, la Ley 388, procuró dejar normas que le permitieran a los municipios regular la construcción y expansión de sus territorios urbanos, la protección de las áreas rurales y ambientales. (Ley 388, 1997)

Según el diagnóstico del eje de vivienda para la revisión y ajuste del POT, en Medellin se tienen cerca de 180 mil hogares ubicados en asentamientos precarios, la mayoría de ellos en las zonas de alto riesgo geológico.

En aras de prevenir posibles desastres naturales que lleguen a costar vidas humanas el municipio en el actual Plan de Ordenamiento Territorial (POT), y acatando las obligaciones legales de la Ley 388 de 1997 se prohíben las obras de urbanismo en estas zonas, las cuales implican necesariamente la instalación de servicios públicos. (Ley 388, 1997)

Reforzando estas normas, la (Decreto 302, 2002) Decreto 302 de 2000 fijo las condiciones de instalación de servicios públicos para acueducto, alcantarillado y gas, restringiendo la obtención de las respectivas licencias de construcción y urbanismo. 
Por otra parte la resolución CREG 108 de 1997 define las condiciones para la instalación de energía eléctrica en la cual se limita al cumplimiento de condiciones puramente formales de capacidades de contratación (Resolusión 108, 1997), sin condicionarla a las características propias del territorio o a la obtención de licencias de urbanismo y construcción, manteniendo las condiciones del artículo 134 de la Ley 142 de 1994: "Del derecho a los servicios públicos domiciliarios. Cualquier persona capaz de contratar que habite o utilice de modo permanente un inmueble, a cualquier título, tendrá derecho a recibir los servicios públicos domiciliarios al hacerse parte de un contrato de servicios públicos”. (Ley 142, 1994)

A estas condiciones se suma que el municipio de Medellín no ha construido un censo catastral de vivienda, es decir, si bien se tiene un censo predial, que se actualizó con la metodología de identificación predial, no hay una caracterización de predio a predio según el uso de cada uno, por lo que se ha recurrido a identificar los predios residenciales de acuerdo a la identificación de uso de cada una de las instalaciones de energía, reportada por los cinco comercializadores de energía activos en la ciudad.

Prestación De Servicios Públicos Domiciliarios En Medellín.

El municipio de Medellín ha construido diferentes diagnósticos de la prestación de servicios públicos domiciliarios, cada uno dependiendo de la fuente de información que se tome puede mostrar resultados diferentes que van desde coberturas del 98 $\%$ en acueducto y del $100 \%$ en energía hasta coberturas de acueducto del $87 \%$.

El Documento Técnico de Soporte (DTS) parte integrante del Acuerdo 46 de 2006 (Acuerdo 046, 2006), sobre servicios públicos se construyó con la información suministrada por Epm, en el cual se calculó una cobertura de acueducto y alcantarillado superior al $98 \%$, y coberturas de energía cercanas al $100 \%$.

Por el contrario el municipio tiene la obligación de cargar la información de coberturas de acueducto y alcantarillado reportado por todas las empresas prestadoras de servicios al Sistema Único de Información (SUI) de la Superintendencia de Servicios Públicos Domiciliarios; según datos de la Subsecretaría de Servicios Públicos de la Alcaldía de Medellín la ciudad tiene en la actualidad 22 empresas prestadoras de servicio de acueducto, y 4 empresas de alcantarillado, incluida Epm en ambos servicios; cuando se toma esta fuente la cobertura de acueducto llega al $87 \%$ en acueducto y al $83 \%$ en alcantarillado. (Epm, 2014) No hay diferencias en la cobertura de energía por dos razones: primero porque, como ya se expuso, el municipio no tiene un censo de viviendas y asume el número de instalaciones residenciales de energía como el número de viviendas $y$, en segundo lugar, la regulación y control del servicio de energía está centrado en los entes nacionales del Ministerio de Minas y Energía, sin darle funciones a los municipios, así se hace desde las medidas que se tomaron luego del racionamiento de 1992.

Estas diferencias de estadísticas se originan en que los datos de Epm, aunque reales, están basados solo en los datos comerciales de la empresa, es decir, dentro de la infraestructura de redes tiene una cobertura superior al $98 \%$, sin que esto signifique que sea igual a la cobertura del municipio, pues queda demostrado con facilidad que hay amplias zonas de la ciudad que no son cubiertas por EPM en ninguno de los servicios domiciliarios que presta la empresa, situación que se 
evidencia en el mapa de cobertura de acueducto elaborado por la Subsecretaría de Servicios Públicos de la Alcaldía de Medellín:

Mapa 1. Cobertura de Acueducto. Medellín.

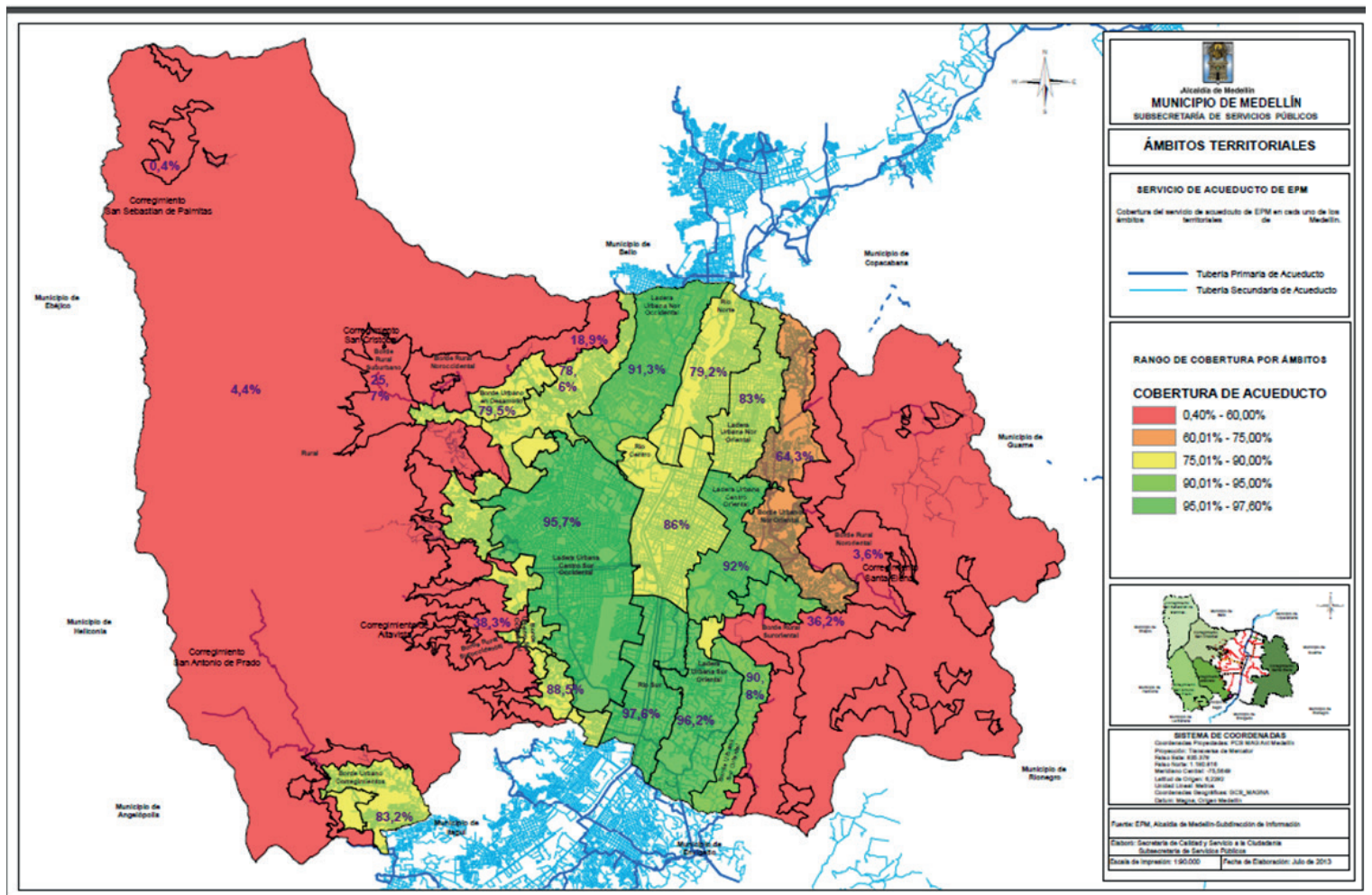

Fuente: Subsecretaría de Servicios Públicos de Medellín, 2013.

Más allá de una discusión de cuál puede ser la fuente estadística correcta la problemática se debe analizar en los términos que señala la Sentencia C-1189 de 2008 de la Corte Constitucional (Sentencia C-1189, 2008) al pronunciarse sobre un artículo de la Ley 812 de 2003 que prohibía hacer inversiones públicas en zonas de alto riesgo no recuperable:

Los servicios públicos han de estar al alcance de todos los colombianos y ninguna norma puede excluir de su acceso a ciertas personas en razón a su condición de pobreza o de marginalidad, como lo hace la norma acusada. Al contrario, el artículo 13 de la Carta señala que la debilidad económica y la marginación deben ser el fundamento de acciones afirmativas en beneficio de quienes por su situación socioeconómica precaria se encuentran expuestos a riesgos, amenazas y vicisitudes que tienen un profundo impacto en su capacidad de llevar una vida digna $y$ de integrarse a las actividades propias de una sociedad organizada. El Estado ha de propender por un crecimiento urbano sostenible y planificado, pero ello no debe hacerse a expensas de excluir del acceso al agua y otros servicios públicos, máxime si aquellos afectados son individuos bajo una situación de especial vulnerabilidad. (Negrillas fuera del texto) (Sentencia C-1189, 2008)

Amparados principalmente en el texto de esta sentencia los jueces de Tutela han seguido protegiendo el derecho de las personas a los servicios públicos. 
Protección del derecho a los servicios públicos mediante vía judicial.

La mayoría de las personas que recurren a la vía judicial para la protección de su derecho a los servicios públicos lo hacen aduciendo como derecho fundamental vulnerado, el derecho a una vivienda digna o el derecho al mínimo vital, en ambos casos la corte los ha protegido por su conexidad con el derecho fundamental a la vida, pero no por ser un derecho en sí mismo, autónomo; sin embargo las sentencias han ido creando una especie de jurisprudencia que permite asegurar los casos especiales en los cuales las cortes siempre van a proteger ese derecho a los servicios públicos.

Presentamos cuatro casos de sentencias de la Corte Constitucional en las que sus pronunciamientos son significativos en materia de protección al derecho a una vivienda digna, como las sentencias Sentencia T-109/11 (Sentencia T-109, 2011) y Sentencia T-725/08. (Sentencia T-725, 2008) Estas dos sentencias se toman como ejemplo de la protección que la justicia hace de las personas que construyen sus viviendas en las zonas de alto riesgo y obliga al Estado a garantizar las condiciones básicas de subsistencia a los hogares en esas circunstancias. Las otras dos sentencias que se analizan: Sentencia T-408/08 (Sentencia T-408, 2008) y Sentencia T-865/11, (Sentencia T-865, 2011) protegen los hogares en la prestación del servicio como tal, protegiendo el derecho a un mínimo vital en materia de servicios públicos.

\section{$\underline{\text { Sentencia T-109/11. }}$}

"Derecho a la vivienda digna", naturaleza y alcance.

Es de concluir, entonces, que una "vivienda digna" debe tener condiciones adecuadas que no pongan en peligro la vida y la integridad fisica de sus ocupantes, pues ella además de ser un refugio para las inclemencias externas, es el lugar donde se desarrolla gran parte de la vida de las personas que la ocupan” (Sentencia T-109, 2011) .

En Medellín, así como en todas las ciudades del país, la población más pobre busca asentarse en las zonas donde tenga menos riesgos de ser expulsados por los organismos de seguridad, donde encuentre redes de solidaridad y apoyo y donde encuentre tierras de más bajo costo para poder construir una vivienda con los menores costos, alli donde la construcción se puede hacer sin cumplir con las mínimas normas del urbanismo, la construcción, ni los materiales adecuados.

Son las construcciones que se encuentran en las zonas de alto riesgo, y de las cuales las personas solo salen por último recurso, o cuando ya ha sucedido una tragedia.

En Medellín la expansión hacia las laderas se ha dado en dos sentidos primordiales:

Primero en la zona suroriental, El Poblado, mediante la construcción de viviendas de estratos altos, con el cumplimiento de las normas básicas de construcción y licenciamiento por parte de curadurias y el recibo de obra por parte de la oficina de Planeación, pero que por ser construcciones altamente costosas el constructor promete cumplir con todas las normas técnicas de mitigación del riesgo y garantizar las condiciones mínimas para el suministro de servicios públicos. Sin embargo las condiciones de licenciamiento hacen que constructores 
busquen mayores rentabilidades económicas por encima de las normas técnicas que en teoria deberían cumplir.

Casos como el desplome de la torre 6 de la unidad residencia SPACE demuestran que a pesar de ser vivienda de estrato alto, la negligencia de funcionarios y/o constructores obligan a que se revise con urgencia los procedimientos, las normas y las condiciones de construcción.

Segundo, el resto de las laderas, los bordes urbanos, se han construido de manera informal, no hay la inversión de grandes constructores haciendo edificios altos de apartamentos y centros comerciales, sino pequeñas viviendas de uno, dos o hasta tres pisos de una misma familia, la mayoría de las veces, no hay licenciamientos de ningún tipo y las conexiones de servicios se hacen casi siempre de manera ilegal.

En el primer caso, los servicios públicos son conectados directamente por la empresa prestadora del servicio, Epm, en el segundo, al conectarse de manera ilegal de la energía Epm, mediante el programa de Habilitación Vivienda (HV), legaliza esas instalaciones, ya sea a través de contadores individuales, pilas públicas o medidores prepago, esto con el fin, de un lado, de evitar las pérdidas que se generan en las conexiones ilegales, y por otro de cumplir con las condiciones de la Ley 302 de 2000 y la resolución Creg 108 de 1997 y de esa manera evitar, las demandas o quejas ante la Superintendencia de Servicios Públicos Domiciliarios; y esperan las Acciones de Tutela para los servicios de acueducto y alcantarillado en cuyas respuestas siempre aducen no ser de su competencia por ser viviendas ubicadas en zonas de alto riesgo o por fuera del perimetro urbano y vinculando en la responsabilidad al municipio obligado por Ley a garantizar la prestación del servicio a todas las personas.

Esta obligación legal de prestación del servicio a todas las personas lleva consecuentemente a que en las zonas de alto riesgo el municipio se vea en la obligación, o de prestar el servicio, o de hacer el consecuente proceso de reubicación, lo que genera altos costos económicos y lleva al municipio a plantearse la necesidad de hacer inversiones para la mitigación del riesgo y pedir a Epm la instalación de servicios, una medida que no siempre es la más acertada en términos sociales y de gestión real del riesgo, pero que es más económica y da mayores réditos políticos en términos electorales.

Sentencia T-725/08 (Sentencia T-408, 2008). Derecho A La Vivienda Digna.

-Es un derecho fundamental cuando se trata de personas desplazadas por la violencia. El enfoque de análisis del caso adquiere otra dimensión en cuanto el predio está ocupado por personas desplazadas por la violencia. La Corte Constitucional ha sostenido que el derecho a una vivienda digna es un derecho fundamental cuando se trata de personas desplazadas por la violencia, y en esos casos especificos es susceptible de ser protegido mediante la acción de tutela. En consecuencia, la Corte ha indicado que es un deber del Estado, en el marco de procesos de desalojo de zonas de alto riesgo, garantizar el derecho a la vivienda digna a la población desplazada victima del(os) conflicto(s) armado(s) interno(s). (Sentencia T-408, 2008). 
En los casos de población desplazada se da una situación muy similar a la anterior, la vivienda digna se reconoce como derecho fundamental en los casos de la población víctima de desplazamiento forzado, no sólo por ser víctimas sino como una estrategia de reparación y atención en el lugar de acogida.

El desplazamiento forzado en Colombia ha adquirido unas dimensiones alarmantes, fruto de la violencia generada por los diferentes grupos armados, tanto legales como ilegales, esta situación ha traído consigo problemas adicionales a las ciudades, pues buena parte de esta población busca asentamiento en las laderas, por las razones que ya arriba habiamos expuesto. Esto obliga necesariamente a que se registre una expansión de la ciudad en los lugares donde las personas necesitan construir sus viviendas y que allí se les garantice la prestación de los servicios públicos domiciliarios.

Sentencia T-408/08 (Sentencia T-408, 2008). Acción De Tutela Contra Empresas Públicas De Medellín.

-Vulneración a la prestación eficiente de los servicios públicos domiciliarios y orden de reubicación por encontrarse la vivienda ubicada en zona de alto riesgo no recuperable Plan De Ordenamiento Territorial-Elementos que debe contemplar Plan De Ordenamiento Territorial-Señalamiento y localización de las áreas de riesgo para asentamientos humanos y estrategias de manejo de las zonas expuestas a amenazas y riesgos naturales. (Sentencia T-408, 2008)

Tal como se ha expresado ya en diferentes apartes el servicio de energía es el que menos restricciones tiene, tanto técnicas como legales, esto facilita la expansión ilegal de viviendas.

Sin embargo llama la atención como Epm sólo esgrime como restricción para la instalación de energía las normas territoriales en algunos casos, en otros procede con la instalación de pilas públicas o medidores prepago.

El sistema de pila pública es un medidor comunitario que suministra la energía para un sector y la empresa factura no por consumos individuales sino por el promedio de consume del estrato de las viviendas conectadas a este medidor.

Los medidores prepago son un sistema implementado por Epm para la recuperación de cartera de los hogares desconectados, en el que con la instalación de un dispositivo electrónico las personas pueden hacer una recarga de energía por mínimo tres mil pesos y el $10 \%$ de esa recarga se destina al pago de la deuda y el resto se le suministra en kilovatios de energía.

Tal como se ha expresado la norma territorial que restringe la instalación de servicios públicos en zonas de alto riesgo no aplica para el servicio de energía, pues al no tener restricciones desde una norma nacional, no tener mayores dificultades de orden técnico Epm procede con esa instalación.

Cuando se ha protegido la prestación del servicio de energia por via judicial se ha hecho al amparo de necesidades que vinculan este servicio directamente con el derecho a la vida, como en el caso de enfermos oxigeno dependientes, aunque en la sentencia analizada no es el caso, la protección de la vida si obliga a que esto sea tenido en cuenta. La otra situación que se presenta es la vinculación al municipio por el tema de 
reubicación de las personas asentadas en zonas de alto riesgo no recuperable. En las sentencias donde no se ordena la prestación del servicio los jueces de Tutela han ordenado la reubicación de la población.

\section{Sentencia T-865/ 11 (Sentencia T-865, 2011). Derecho A La Vivienda Digna.}

-Fundamental por conexidad o por afectación del minimo vital. El concepto de vivienda digna implica contar con un lugar, propio o ajeno, que le permita a la persona desarrollarse en unas minimas condiciones de dignidad y satisfacer su proyecto de vida. Igualmente, el artículo 51 de la Constitución Política consagra el acceso a una vivienda digna como un derecho de todas las personas, y asigna al Estado la obligación de fijar las condiciones necesarias para hacerlo efectivo a través de la promoción de planes de vivienda de interés social, sistemas adecuados de financiación a largo plazo y formas asociativas para la ejecución de dichos programas. (Sentencia T-865, 2011)

Medellín fue la primera cuidad del país en contar con un programa social denominado Mínimo Vital de Agua Potable. Según se establece en el Acuerdo 06 del 25 de abril de 2011 y en el Decreto Municipal 1889 de noviembre de 2011 este programa consiste en el descuento del valor de 2,5 metros cúbicos de acueducto y alcantarillado por persona en el hogar; el Decreto 013 de 2014 modificó la restricción que existia de que los hogares tenían que hacer parte de un programa de asistencia y acompañamiento familiar denominado Medellín Solidaria o al programa del gobierno nacional Familias en Acción o registradas como población desplazada y le da mayor cobertura al tener como único requisito el cumplir con tener un puntaje en la encuesta del SISBEN inferior a 47,99.

Este programa tiene algunas dificultades principalmente las siguientes:

Funciona en forma de un descuento en la factura del consumo, luego los hogares que tienen los servicios suspendidos no verán reflejado este beneficio pues no se genera factura sobre la cual puedan ver efectivamente aplicado le descuento al que tienen derecho... Aunque se ha superado la restricción de pertenecer a Medellin Solidaria o Familias en Acción los hogares manifiestan la inconsistencia de los puntajes del SISBEN, pues no se ven con claridad la objetividad de esta encuesta. (Sentencia T-865, 2011)

El Mínimo Vital se ha vuelto en las sentencias de la Corte Constitucional un tema de principal relevancia frente a los temas de servicios públicos domiciliarios, sobre porque este concepto ha ido creando la jurisprudencia necesaria para ser considerado un derecho fundamental.

Se ha reconocido desde la Corte como Mínimo Vital el derecho inalienable de la población especial y constitucionalmente protegida: menores de edad, adulto mayor, personas en situación de discapacidad, madres gestantes, personas con enfermedades graves, degenerativas o terminales y madres solas cabeza de familia, incluso se ha ordenado a las empresas prestadoras de servicios públicos que en los casos en los cuales se verifica pro parte de un funcionario de la empresa que en la dirección reside una persona que cumpla con una de estas condiciones no se podrá suspender definitivamente el servicio de agua, ni el de energía si hay personas que requieran de ella para el mantenimiento de su vida. 
Estas sentencias que dan este tipo de órdenes nunca se ha respetado por parte de EPM, por ausencia de pago en dos facturas continuas se suspende el servicio y se espera que la persona afectada recurra a la Tutela o la conexión ilegal o fraudulenta, situación por la que algunos jueces han negado la protección de Tutela.

Es desde la concepción del Mínimo Vital que se habla de los Servicios Públicos Domiciliarios como un derecho fundamental, pues son elementos indispensables para el desarrollo y el mantenimiento de la vida en condiciones mínimas de dignidad.

Cuando se aborda el tema de los servicios públicos domiciliarios desde una perspectiva de los derechos humanos es preciso tener en cuenta que estos no son derechos en sí mismos, sino que la ausencia de estos servicios vulnera todos los demás derechos fundamentales, al impedir el desarrollo, limitar la dignidad, imposibilitar el acceso a oportunidades para el mejoramiento de la calidad de vida.

Es en las sentencias de la Corte Constitucional en las que se puede dar respuesta al título inicial del presente artículo: Servicios Públicos Domiciliarios: ¿Derechos Humanos o Productos Para la Venta? Pues es a través de ellas que podemos identificar que a pesar del tratamiento comercial que se le da a la regulación de prestación de servicios, de primar los análisis económicos, financieros y de rentabilidad para determinar la viabilidad o no de prestación del servicio, el reconocimiento de la necesidad humana de contar con estos servicios como elemento fundamental para el desarrollo les da el carácter de un derecho humano fundamental.

\section{Conclusiones y recomendaciones.}

Alternativas a la mercantilización de los servicios públicos domiciliarios:

Si bien es claro que el actual esquema de prestación de servicios públicos domiciliarios está basado en una concepción empresarial y por lo tanto de rentabilidad económica no sería posible plantear una alternativa a la mercantilización desde ese mismo enfoque.

Es claro que cualquier posibilidad que brinde reconocimiento de derechos humanos fundamentales a la prestación de los servicios públicos domiciliarios tiene que pasar, necesariamente, por un cambio radical del actual esquema, y por lo tanto modificar la Ley 142 de 1994.

Los actuales fallos judiciales han reconocido el derecho a los servicios públicos y al suministro del mínimo vital, pero esto no consulta con las capacidades económicas de la empresa, ni de la población misma, y el fallo obliga la prestación del servicio, pero no determina la obligación del pago de ese servicio en cabeza de quien recae. El argumento de las empresas es que si a todo fallo judicial se obliga la prestación en forma gratuita se pone en riesgo la estabilidad económica de la empresa, por otra parte se genera un vacío y una contradicción con la misma Ley 142 de 1994, pues ella en su artículo 99 numeral 9 dice:

Los subsidios que otorguen la nación y los departamentos se asignarán, preferentemente, a los usuarios que residan en aquellos municipios que tengan menor capacidad para otorgar subsidios con sus propios ingresos. En consecuencia y con el fin de cumplir cabalmente con los principios de solidaridad y redistribución 
de ingresos no existirá exoneración en el pago de los servicios de que trata esta Ley para ninguna persona natural o jurídica. (Ley 142, 1994)

Sin embargo, y quizás como en la canción de Fito Páez:

"Quién dijo que todo está perdido

Yo vengo a ofrecer mi corazón

Tanta sangre que se llevó el río

Yo vengo a ofrecer mi corazón"

Las mismas comunidades han ido creando alternativas, unas, como en la canción, ofreciendo el corazón en todo lo que hacen, otras mediante el uso y el abuso de la fuerza, algunas de ellas son:

Unas desde la perspectiva de la resistencia legal y jurídica a los abusos de una multinacional, entre ellas la Mesa Interbarrial de Desconectados, asesorando las comunidades en la defensa de sus derechos mediante la vía judicial, interponiendo las acciones de Tutela y las Acciones Populares, denuncias internacionales y eventos de protesta pacífica frente a los edificios de la administración municipal y de Epm.

En el libro electrónico "Alternativas a la Privatización: la provisión de servicios públicos esenciales en los países del sur" los autores David A. McDonald y Greg Ruters presentan algunas dificultades que se han tenido en los modelos privatizadores, no solo desde el esquema de las empresas privadas, sino del esquema que, como en Colombia, se implementa con las empresas industriales y comerciales del Estado, ellos afirman:: "La propiedad estatal (es decir, pública), por ejemplo, puede servir a los intereses de las élites y las grandes empresas y marginar a los pobres". (McDonald \& Ruiters, 2012)

Esas alternativas que se presentan como experiencias exitosas son precisamente aquellas que no se fundamentan en los esquemas comerciales, sino en las iniciativas comunitarias constituyéndose en otra posibilidad legal que conforman las 21 empresas de acueducto veredal existentes en la ciudad y las 4 que tienen sistemas de alcantarillado. Estos sistemas comunitarios y veredales, cuentan con el acompañamiento y asesoría técnica, comercial y jurídica por parte del Municipio de Medellín; estos sistemas en muchos casos son incluso anteriores a la misma Epm y es la organización social y comunitaria que las ha defendido y han sido un factor de cohesión social en las comunidades.

Como tercera vía la misma Ley 142 y el Decreto reglamentario 1429 de 1995 que faculta a las comunidades para la conformación de los Comités de Desarrollo y Control Social en Servicios Públicos, estos se crean como veedurías ciudadanas para la fiscalización y el control social a la prestación de los servicios públicos domiciliarios. En Medellín existen 30 de estos comités, registrados ante la alcaldía municipal, pero sólo 16 están realmente activos y su capacidad real de acción se ha limitado, en algunos casos, a brindar capacitación a las comunidades en el uso racional y eficiente de los servicios públicos domiciliarios, pero sin ninguna incidencia real en el esquema de prestación del servicio.

Una cuarta opción se ha vivido en los barrios, esta no legal, ni de organización comunitaria, el conflicto y las organizaciones armadas han encontrado que al controlar el agua controlan también el territorio, la comunidad, el comercio y todo 
cuanto se mueva en el sector. Los "combos", mediante la intimidación y la amenaza, se han hecho elegir en las juntas directivas de las juntas de acción comunal y de las juntas de acueducto. Deciden a quienes desconectan del servicio, por cuanto tiempo y fijan las tarifas. Sobre estas prácticas se han interpuesto denuncias ante la Defensoría del Pueblo y la Personería de Medellín, especialmente de los sectores de La Honda y la Cruz en comuna 3, Llanaditas y Golondrinas en comuna 8, Picacho en comuna 6, El Salado en Comuna 13, Carpinelo y Carambolas en Comuna 1.

Otra opción de resistencia también ha sido la amenaza a los funcionarios de EPM encargados del corte de servicios y de la distribución de la facturación, zonas de la ciudad donde estos funcionarios no pueden ingresar debido a las amenazas constantes y en algunos casos a la retención de su personal.

Una última opción, es la conexión ilegal o fraudulenta mediante las conexiones "directas", es decir, pasando directo de la red principal a la red domiciliaria, sin que se registre en el medidor, la alteración de los medidores para que no registren los consumos reales, o la reconexión manual e ilegal, aunque siga pasando por el contador pero sin la autorización del prestador en los casos en que éste había realizado un procedimiento de suspensión del servicio por falta de pago.

Las tres últimas alternativas presentadas no se pueden pensar como viables en el desarrollo de la ciudad ni en la construcción de una visión de los derechos humanos a los servicios públicos, sin embargo no se puede desconocer que son una práctica real y recurrente en la ciudad de Medellín.

Medellín, la "ciudad más innovadora del mundo", una de las ciudades mejor iluminadas del mundo, el municipio propietario de una de las Empresas de Servicios Públicos Domiciliarios más grandes de América Latina, una de las empresas Multilatinas de mayor reconocimiento, es al mismo tiempo ciudad de escenarios tan desoladores como el tener para 31 de agosto de 2014 un total de 26.515 hogares con el servicio de agua suspendido o cortado, 15.730 en energía y 14.723 de gas; así lo informa la Subsecretaría de Servicios Públicos de Medellín. (Epm, 2014)

El esquema de prestación de servicios públicos domiciliarios como productos para la venta que impera en el país hace que ese fenómeno de la desconexión sea constante y recurrente, que la misma empresa no tenga alternativas viables para la continuidad del servicio más allá del esquema prepago que sólo garantiza la continuidad mientras el usuario tenga los recursos para hacer las recargas constantes, y el municipio sólo crea un programa de asistencia social que permite un descuento en la factura funcionando más como un mecanismo de prevención de la desconexión que como una garantía real de suministro del mínimo vital bajo cualquier circunstancia.

Estas afirmaciones anteriores llevan a poder concluir que los servicios públicos domiciliarios requieren con urgencia un tratamiento distinto en las normas, deben ser considerados bienes estratégicos para el desarrollo, pues de ellos depende no solo la producción, sino la existencia misma de las personas.

Mientras en los barrios no se solucionen y no se brinde con plena garantía el suministro de un mínimo vital de agua y de energía y no se reconozca el derecho fundamental de las personas a gozar en su lugar de residencia de energía y 
agua potable y un sistema de saneamiento básico, las situaciones que originan la desconfianza popular en el Estado, la conformación de formas violentas de organización y control social no se podrán detener.

Hoy, ante la expectativa de un posible escenario de post conflicto, ante el eventual acuerdo de paz con la guerrilla, los temas de conversación tocan los temas que dieron origen $\mathrm{y}$ han agudizado el conflicto: tierras, narcotráfico, participación politica; pero los problemas que se manifiestan en las ciudades y que originan que aquellos entrenados militarmente y formados politicamente para ser poder local no se contemplan, cómo manejarlos en los micro territorios especialmente alli donde desde ya son grupos al margen de la ley quienes controlan y definen la prestación de los servicios, las normas de construcción, etc. Son retos nuevos para una ciudad que no está preparada más que para seguir anunciando con bombos y platillos que pasamos del miedo a la esperanza y que somos la ciudad más innovadora del mundo. 


\section{Referencias Bibliográficas:}

Acuerdo 046, Plan de Ordenamiento Territorial, Medellín. (Acuerdo Municipal. Medellín. 02 de 08 de 2006).

Atehortua Rios, C. (2003). Servicios Públicos domiciliarios: legislación y Jurisprudencia. Medellín: Biblioteca Jurídica Diké.

Decreto 302, Por el cual se reglamenta la Ley 142 de 1994, en materia de prestación de los servicios públicos domiciliarios de acueducto y alcantarillado (Presidencia de la República de Colombia 25 de Febrero de 2002). Obtenido de alcaldiabogota. gov.co: http:/ /www.alcaldiabogota.gov.co/sisjur/normas /Norma1.jsp?i=4636

Epm. (31 de Agosto de 2014). Nuestros Servicios Epm. Obtenido de Epm.com.co: http:// www.epm.com.co/site/clientes_usuarios/Clientesyusuarios/Nuestrosservicios.aspx

Insuasty Rodriguez, A. (2013). Ante la actual crisis de sentido, es necesario "rescatar al sujeto". El Agora USB, 280-290.

Insuasty Rodriguez, A., \& Villa Holguin, E. (2013). Capital Sujeto y ciudad. Lecturas de ciudad y crisis humanista. Caso Medellín. El Agora USB, 87-96. Obtenido de http://web.usbmed.edu.co/usbmed/elagora/htm/v14nro1/pdf/CAPITALSUJETO-Y-CIUDAD-ALFONSO-EDISON.pdf

Ley 142, Por la cual se establece el régimen de los servicios públicos domiciliarios y se dictan otras disposiciones. (Congreso de la República de Colombia 11 de Julio de 1994). Obtenido de acueducto.com.co: http://www.acueducto.com.co/ wpsv61/wps/html/resources/empresa/LEY142DE1994.pdf

Ley 143, Por la cual se establece el régimen para la generación, interconexión, trasmisión, distribución y comercialización de electricidad en el territorio nacional, se conceden unas autorizaciones y se dictan otras disposiciones en materia energética. (Congreso de la Repúbica de Colombia 12 de Julio de 1994). Obtenido de minminas.gov.co: http://www.minminas.gov.co/ documents / 10180/667537/Ley_143_1994.pdf/c2cfbda4-fe12-470e-9d3067286b9ad17e

Ley 1450, Por la cual se expide el Plan Nacional de Desarrollo, 2010-2014 (Congreso de la República de Colombia 16 de Junio de 2011). Obtenido de sinergia.dnp. gov.co: https://sinergia.dnp.gov.co/SISMEG/Archivos / PND2010-2014\%20 Tomo\%20I\%20CD.pdf

Ley 388, Por la cual se modifica la Ley 9 de 1989, y la Ley 2 de 1991 y se dictan otras disposiciones. (Congreso de la República de Colombia 18 de Julio de 1997). Obtenido de minminas.gov.co: http:/ /www.minminas.gov.co/minminas / downloads / UserFiles / File/ENERGIA / fondos\%20especiales / FOES / Ley_388_ de_1997.pdf 
Ley 388, Por la cual se modifica la Ley 9 de 1989, y la Ley 2 de 1991 y se dictan otras disposiciones (Congreso de la República de Colombia 18 de 07 de 1997).

McDonald, D., \& Ruiters, G. (2012). Alternativvas a la Privatización: La provisión de Servicios Públicos Esenciales en los países del sur. . Barcelona: Icaria Editorial S.A.

Resolusión 108, Por la cual se señalan criterios generales sobre protección de los derechos de los usuarios de los servicios públicos domiciliarios (Creg 3 de julio de 1997). Obtenido de apolo.creg.gov.co/: http://apolo.creg.gov.co/Publicac. 